Post Operative Chylothorax Development is Associated with Increased Incidence and Risk Profile for Central Venous Thrombosis

\author{
Michael Andrew McCulloch \\ Charlottesville, Virginia
}

\begin{abstract}
MD, University of Texas Health Sciences Center in San Antonio, 2001 BS, Wake Forest University, 1995
\end{abstract}

A Thesis presented to the Graduate Faculty of the University of Virginia in Candidacy for the Degree of

Master of Science

Department of Public Health Sciences

University of Virginia

May, 2007

Mark Conaway

Viktor Bovbjerg

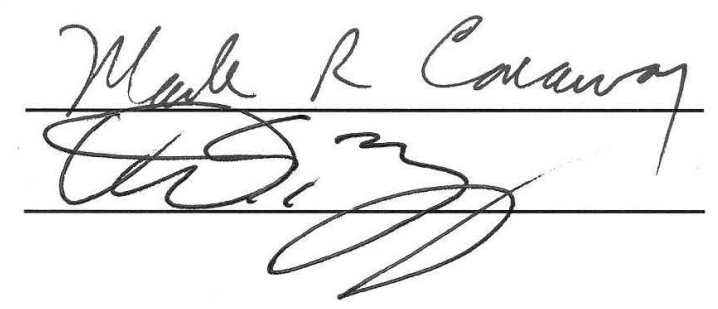




\section{Post Operative Chylothorax Development is Associated with Increased Incidence and Risk Profile for Central Venous Thrombosis}

Background: Chylothorax (CTX) is a complication reported to occur postoperatively in up to $4 \%$ of all pediatric patients requiring surgery for congenital heart disease. It has been associated with prolonged ventilator dependence, increased length of hospital stay, malnutrition, infection, and death. The available treatment modalities are either ineffective, associated with substantial risk, or both. Prevention of CTX appears to be the best option.

Objective: We hypothesized that pediatric patients who develop CTX after surgery for congenital heart disease have an elevated incidence and risk profile for central venous thrombosis (CVT).

Design/Methods: A retrospective chart review was performed of 30 patients found to have developed CTX at the University of Virginia after surgery for congenital heart disease. All but one CTX patient were surgery, anatomy, and age matched with two controls (NON-CTX) to compare their relative risk and incidence of CVT.

Results: Using conditional logistic regression analyses, CTX development was associated with significantly longer ventilator dependence $(14.8 \pm 10.9$ vs. $6.1 \pm 5.9$, $\mathrm{p}=0.003$ ) and a non-significant trend towards more days of central venous catheters (CVC) $(19.1 \pm 16.6$ vs. $12.2 \pm 10.0$ days; $\mathrm{p}=0.16)$ when comparing the period prior to CTX development in the CTX group with the entire hospitalization in NON-CTX patients. CTX development was also associated with a significantly elevated mortality risk (6.2; 95\% CI 1.3-30.9). Minimum, maximum, and average daily central venous pressures were 
significantly higher in the CTX group. Post operative need for extracorporeal membrane oxygenation conferred an increased risk of CTX development in this sample of patients (9.9; 95\% CI 2.2-44.8). Incidence of documented CVT was 26.7\% in the CTX group verses $5.1 \%$ in the NON-CTX group.

Conclusions: CTX portends a significantly worse outcome in pediatric patients who are status post surgery for congenital heart disease. Prospective screening for CVT risk and formation, combined with early removal of CVCs may help reduce the incidence of CTX. 


\section{Background}

Chyle is a milky fluid that courses through the lymphatic system. Its milky character is due to triglycerides composed of long chain fatty acids which are absorbed from the intestines directly into the lymphatics. In addition to absorbing these triglycerides, one of the major roles of the lymphatic circulation is to carry the cells of the immune system from lymph nodes to the blood and on to body tissue. The lymphatics course throughout the entire body, eventually draining into a larger vessel called the thoracic duct which typically drains into the left subclavian vein and into the heart (Figure 1).

Drainage of the chest cavity by the lymphatic system comprises another of its most important roles. If the lymphatic system is unable to absorb this chylous fluid from the pleural space, a chylothorax (CTX) will develop. In the scientific literature, this complication is reported to occur in up to $4 \%^{1-5}$ of all pediatric patients requiring surgery for congenital heart disease and is associated with prolonged ventilator dependence, longer hospital stays, repeat surgeries, malnutrition, increased infection rates, and death ${ }^{5-}$ 11.

While malignancy, congenital lymphatic malformations and direct surgical trauma to the thoracic duct are the most commonly sited causes of CTX in the adult scientific literature $^{12-15}$, it has been our experience that most cases of CTX in the post-operative pediatric cardiac surgery patient are idiopathic in origin. It is plausible that these idiopathic cases are associated with central venous thromboses and the resultant venous congestion (central venous hypertension). Normal lymphatic drainage into the central venous system requires relatively low central venous pressure. In the presence of central 
venous hypertension, this normal flow is disrupted and the thoracic duct becomes engorged. Hydrostatic pressure within the thoracic duct begins to exceed that of the pleural space and chylous fluid extravasates into the chest cavity, forming a CTX. Therefore, any cause of central venous hypertension, including central venous thromboses, may lead to CTX formation.

The neonate and older child with congenital heart disease have been shown to be deficient in important enzymes of the anti-coagulation pathway both before ${ }^{8}$ and after surgery requiring cardiopulmonary bypass ${ }^{8,16-21}$. In one study, nearly $50 \%$ of all infants less than six months of age and $30 \%$ of children older than six months of age diagnosed with a central venous thrombosis also had an underlying cardiac disorder ${ }^{18}$; the etiology of this association is unclear. One study addressing this topic retrospectively evaluated 1591 pediatric patients requiring cardiac surgery with cardiopulmonary bypass ${ }^{8}$. Approximately $1.1 \%$ of these patients developed central venous thromboses with an associated mortality rate of nearly 40\%; a deficiency of the anti-clotting enzyme Antithrombin III was present in $25 \%$. In contrast, in a separate cohort of 1086 pediatric patients undergoing cardiac surgery not requiring cardiopulmonary bypass, only $0.2 \%$ developed central venous thromboses ${ }^{8}$. Subgroup analysis of the cardiopulmonary bypass-requiring cohort demonstrated neonates to have a ten-fold increase in central venous thrombosis incidence when compared with older children.

Recently published data in this field prospectively evaluated Antithrombin III activity in ten children who developed CTXs after cardiopulmonary bypass-requiring cardiac surgery ${ }^{20}$. When compared to five patients with non-chylous pleural effusions, the 
authors demonstrated significantly lower serum Antithrombin III activity and higher pleural effusate Antithrombin III activity in the CTX patients. Further, seven of the CTX patients were diagnosed with central venous thrombosis; only three of these central venous thromboses were supracardiac in location.

Presumably, the anatomic location of the central venous thrombosis would be critical when considering its causative role in CTX development. To disrupt the prograde flow of the lymphatic circulation into the central venous circulation, thrombosis would seemingly need to exist between the heart and the insertion of the thoracic duct into the left subclavian vein. Only then should the resultant central venous hypertension be 'transmitted' to the thoracic duct. However, this may not be the only mechanism through which thromboses can alter lymphatic flow. The role of embolic events in pulmonary hypertension has been well documented ${ }^{22}$ and this state can increase right ventricular, right atrial, and ultimately central venous pressures. Both the wide array of normal lymphatic anatomy and the impaired accessory lymphatic channel function in the presence of infracardiac central venous thrombosis minimize the necessity of supracardiac central venous thrombosis anatomy in the development of CTX.

Regardless of whether this coagulopathy is congenital or acquired, when coupled with the central venous catheter present in nearly all post-operative cardiac surgery patients, the risk of thrombus formation increases significantly. Virchow's Triad states that vascular endothelial damage, a hypercoagulable state and abnormal blood flow all independently predispose to vascular thrombosis ${ }^{23}$. Endothelial injury to the blood vessel can be induced by the mere placement and continued presence of a central venous 
catheter. The hypercoagulable status concurrent in much of this patient population further increases the risk of thrombus formation ${ }^{23}$. Lastly, a low flow state develops in the setting of decreased cardiac output, anatomical obstruction, or pulmonary hypertension; all of which are frequent post-operative complications of surgery for pediatric congenital heart disease. Though there are no data evaluating the incidence of central venous thrombosis in this particular patient population, under these circumstances, it is likely quite high.

Definitive treatment for CTX is lacking and constitutes the major complication with this disease state. Initial conservative treatment regimens focus on reducing the production of chyle by removing triglycerides from the diet. This is generally accomplished using medium chain triglycerides (MCTs; e.g. Monogen ${ }^{\circledR}$, Portagen ${ }^{\circledR}$; medium chain triglycerides are not absorbed into the lymphatic system) as the only source of dietary fat ${ }^{24}$ or through continuous fasting with only parenteral nutrition. However, these regimens are very difficult to maintain for the standard minimum of two weeks and the central venous catheters needed to administer intravenous nutrition have been associated with a $13-26 \%$ rate of central venous thrombosis formation ${ }^{17,18,25}$. Further, parenteral and enteral MCT therapies have been shown to be only $75 \%$ and $33 \%$ effective, respectively ${ }^{26}$.

The only published medical intervention is octreotide, the long-acting, synthetic analog of the endogenous hormone somatostatin; evidence for its efficacy is limited to case studies $^{1-3,10,27-31}$. Like somatostatin, octreotide is a potent inhibitor of glucagon, insulin, and growth hormone ${ }^{26}$. In addition, octreotide affects the gastrointestinal system by decreasing splanchnic blood flow and inhibiting secretion of the hormones gastrin, 
serotonin, secretin, motilin, and vasoactive intestinal peptide ${ }^{26}$. The exact mechanism through which octreotide therapy affects chylous fluid production is unknown. It has been postulated that reduced thoracic duct flow is secondary to the reduction in splanchnic blood flow with a subsequent decrease in triglyceride absorption ${ }^{32}$. Further complicating the use of this medication is the absence of any randomized or controlled trials evaluating dosing strategies or routes.

When conservative measures prove unsuccessful, invasive strategies are commonly employed. Chest tube placement is the most common intervention used to drain the chylous fluid. When a CTX proves refractory to the above treatments, prolonged periods of drainage can result in severe protein loss, malnutrition, and a suppressed immune system. Under these circumstances, a pleuro-peritoneal or Denver ${ }^{\circledR}$ shunt can be inserted. These surgically placed, long-term, subcutaneously tunneled chest tubes drain the chylous fluid accumulation from the pleural cavity to the abdominal cavity where it can be reabsorbed ${ }^{11,33}$. Both of these treatments are associated with significant risks of infection and malfunction, typically requiring surgical replacement and antibiotic therapy.

The remaining treatment options are ligation of the thoracic duct at its inferior origin into the thoracic cavity ${ }^{11,33-36}$ or chemical pleurodesis ${ }^{9}$. Thoracic duct ligation requires a midline sternotomy with chest exploration and carries a risk of hemi-diaphragm paralysis and infection; even a 'successful' ligation can be a clinical failure, however, due to the wide anatomic variability of the lymphatic system. Chemical pleurodesis, or chemical 'scarring' of the lung to the chest wall, precludes further intervention within the pleural 
cavities and presents a technical quagmire to future surgeons entering the chest; anecdotally, success rates are poor. Overall, no optimal therapeutic option exists to treat this state and for the options that do exist, there is limited data as to their effectiveness. Prevention is therefore the best available alternative.

Due to the paucity of data existing on chylothorax etiology, risk factors, and management in the pediatric post-operative cardiac surgery patient, we performed a retrospective analysis of factors potentially contributing to and/or associated with CTX in pediatric congenital heart surgery patients. The hospital course of all children diagnosed with CTX at the University of Virginia between January 1999 and January 2007 were analyzed. We hypothesized that pediatric patients who develop CTX after surgery for congenital heart disease have a worse risk profile for and an increased incidence of central venous thrombosis (CVT) when compared with anatomy, surgery, and age matched patients who do not develop CTX. 


\section{$\underline{\text { Materials and Methods }}$}

After obtaining approval by the Investigational Review Board, the University of Virginia's Pediatric Intensive Care Unit (PICU) database was queried for all patients who underwent surgery for congenital heart disease, starting in January 1999. The search was narrowed for those who also developed a pleural effusion and then for those who were diagnosed with a chylothorax. In addition, the University of Virginia's Pediatric Pharmacy produced a list of inpatients receiving octreotide therapy. This combined search resulted in 30 such patients diagnosed with chylothorax of which nine were treated with octreotide. The University of Virginia's Pediatric Cardiology database was then searched in an effort to surgery, anatomy, and age-match each chylothorax (CTX) subject with two, non-chylothorax (NON-CTX) controls. In the event of multiple matches, the CTX subject was paired with NON-CTX controls that underwent surgery within the same year. When exact NON-CTX matches were not available for CTX patients with complex single ventricle physiology and anatomy, subjects were matched with controls who had undergone similar operative procedures. Further, four biventricular patients with a combination of Tetralogy of Fallot and Atrioventricular Canal Defects (TOF/AVC) were repaired during the study period of which three developed CTX. The remaining five NON-CTX matches were comprised of subjects who underwent repair of AVC (4) and TOF (1) defects alone. This search process produced 59 NON-CTX controls (data was available for only one NON-CTX subject status post neonatal heart transplant).

Demographic data included age at admission and surgery, weight on admission, original cardiac anatomy and surgical intervention. When applicable, total cardiopulmonary bypass (CPB) time was recorded. Duration of Intensive Care Unit 
(ICU) and total hospital stay were also collected. Daily flow sheets were examined for the entire hospital course to collect the following data: periods of no enteral feedings (NPO), periods of Total Parenteral Nutrition (TPN), periods of low fat or MCT formula use, periods of ventilator dependence, periods and location of central venous catheters (CVC), periods of Extracorporeal Membrane Oxygenation (ECMO), periods of octreotide therapy, daily volumes and origin of chylous fluid drainage, and the daily ranges of central venous pressures (CVP) in $\mathrm{mmHg}$. The daily progress notes were evaluated in the event of uncertainty in the daily flow sheets. When overlap of two opposing periods (i.e. NPO and low fat diet) occurred during a hospital day, the day was credited to the entity lasting greater than 12 hours. The University of Virginia's clinical data repository was searched for chest radiograph frequency and the following laboratory data: serum white blood cell count (WBC), total protein, albumin, triglycerides (TAG), and Antithrombin III activity (ATIII), as well as pleural WBC, percentage of lymphocytes, and TAG content. The clinical data repository was also evaluated for all fluoroscopy, ultrasound, MRI, CT, cardiac catheterization, and interventional radiology results to determine the incidence of documented CVT.

The date of CTX diagnosis was determined by both a change in medical management (i.e. addition of octreotide therapy, change to NPO status, change to low fat diet, etc.) and the initial inclusion of the diagnosis "chylothorax" in the assessment and plan section of the attending physician's daily progress note. All but two CTX subjects were diagnosed and treated at our institution after placement of either a pleural drainage tube $(n=27)$ or thoracentesis alone $(n=1)$. One CTX subject who received surgical intervention at our 
institution was ultimately diagnosed with CTX at an outside hospital prior to being transferred back for further management and a second CTX subject was started on MCT formula two days prior to the removal of pleural fluid which was chylous.

\section{$\underline{\text { Statistical Methods }}$}

To account for the 2:1 matching of NON-CTX to CTX patients, conditional logistic regression was used for analysis. Multivariate conditional logistic regression analysis was performed using the continuous variables of CVC duration and CVP values prior to CTX diagnosis and the binary variable of need for ECMO prior to CTX diagnosis. These variables were chosen a priori to evaluate their association with the development of CTX. Statistical significance was defined as $\mathrm{p}<0.05$. All statistics were performed using the SAS statistical package (Cary, NC). 
$\underline{\text { Results }}$

Age at admission and surgical intervention did not differ statistically between the CTX and NON-CTX groups, though the median age was approximately two months older in the NON-CTX group as shown in Table 1. Weight on admission was also not significantly different between the groups. CTX patients were ventilator dependent approximately seven times longer than NON-CTX patients; this difference remained significant when comparing entire hospitalization NON-CTX ventilator dependence to ventilator dependence prior to diagnosis of CTX in the CTX group (14.8 \pm 10.9 days (median 13.0); $\mathrm{p}=0.003$ ). CTX patients received four times as many CXRs and required significantly longer periods of NPO and HAL dependence. CTX subjects had significantly longer ICU and total hospitalization durations as well as a nearly four fold increase in total cost as compared with NON-CTX patients.

The median number of post-operative days until CTX diagnosis was 11.5 (average 13.4 \pm 8.2 ). CTX patients had a six fold increased mortality risk when compared with the NON-CTX group.

Table 2 demonstrates the original cardiac anatomy of the CTX group. Over the study period, a total of 650 surgeries performed at our institution were similar to those outlined in this table, including these 30 patients; the resulting incidence of CTX was $4.6 \%$. This incidence increased to $5.8 \%$ when considering only patients with single ventricle physiology and anatomy.

Of the 30 patients with CTX, eight had effusions from the left pleural space, three from the right pleural space, 16 demonstrated bilateral pleural effusions, one had peritoneal 
fluid only, and two patients produced chylous fluid from the peritoneal cavity and both pleural spaces.

Univariate, conditional logistic regression demonstrated a ten fold increased risk of CTX development in patients who required ECMO in the immediate post-operative period, as shown in Table 3. Matched CVPs were significantly higher for both the minimum and average values and trended towards significant for maximum daily values. Further, the CTX group had CVCs in place five and six days longer in their upper and lower extremities, respectively, than the NON-CTX group; this difference did not reach statistical significance. It is important to note that the CVC and CVP data in Table 3 are all prior to CTX diagnosis in the CTX group as compared to the entire hospitalization for the NON-CTX group.

Table 4 demonstrates the average values of each lab either on or one day prior to the day of CTX diagnosis.

The lab values in Table 5 demonstrate the cumulative average of total protein, albumin, serum triglycerides, and serum white blood cell count in CTX subjects prior to and after the date of CTX diagnosis. Although there were statistically significant differences in serum albumin and white blood cell counts pre and post CTX diagnosis, these differences were not clinically meaningful. ATIII activity was not obtained enough to allow for statistical analysis.

Multivariate conditional logistic regression analysis was performed using the continuous variables of total CVC duration (including both upper and lower extremity locations) and average CVP values prior to CTX diagnosis and the binary variable of 
need for ECMO prior to CTX diagnosis as compared with the entire hospitalization for the NON-CTX group. This constellation of variables were significantly different between the CTX and NON-CTX groups $(\mathrm{p}<0.0001)$. Only elevated average CVP values independently increased CTX risk with an Odds Ratio of 1.59 (95\% CI 1.30-2.40).

There were eight documented cases of CVT in the CTX group (26.7\%) of which five were supracardiac in location, one was infracardiac in location, and two patients had CVTs in both locations; four of these patients had single ventricle physiology and anatomy. The NON-CTX group had three documented cases of CVT (5.1\%) of which one was infracardiac in location and two were supracardiac in location; all three patients had single ventricle physiology and anatomy.

No meaningful patterns were demonstrated with regards to the effect of octreotide, MCT only diet, or NPO management on daily volumes of chylous chest tube output. As a result, the effectiveness of these strategies was not amenable to statistical analysis in this study. 


\section{$\underline{\text { Discussion }}$}

To our knowledge, this is the first controlled study to demonstrate an increased incidence of and risk profile for CVT in pediatric patients who developed CTX after surgery for congenital heart disease. CTX was also associated with significantly more radiation exposure, longer periods of HAL and ventilator dependence, longer intensive care unit and overall hospitalization stays, and markedly higher costs.

A link between CVT's and CTX development has been suggested in reviews, case reports and case series ${ }^{6,14,37-40}$. Dhande ${ }^{38}$ described five neonates with CTX that followed SVC obstruction from CVT. Others have published their experience with CTX after CVT development associated with septic shock ${ }^{37}$ and a $\mathrm{CVC}^{40}$. Manghat ${ }^{14}$ reported resolution of chylous effusions after an extensive upper extremity CVT was successfully reduced with thrombolysis in an adult patient with Down's syndrome and lymphoblastic leukemia. In the only study comparing chylous to non-chylous effusions, BernetBuettiker $^{20}$ found activity of the anticoagulation enzyme ATIII to be significantly increased in chylous effusions and decreased in the serum of ten pediatric patients after surgery requiring $\mathrm{CPB}$ in comparison to five such patients with non-chylous pleural effusions.

While less than $27 \%$ of the CTX group in our study had documented CVTs, they exhibited a significantly increased risk profile for CVT formation as compared with the NON-CTX group. In our analysis, we considered all post-operative cardiac surgery pediatric patients to be at risk for CTX development with an end point of either CTX diagnosis or discharge from the hospital. As such, those in the CTX group had 
significantly more exposure to ECMO, an average of five to six days longer exposure to CVCs, and significantly higher CVP (minimum and average) than those who did not develop CTX prior to hospital discharge. When combined with the assertion that pediatric patients with congenital heart disease have an increased prevalence of genetic coagulopathies $^{6,18,21}$, those in the CTX group met each of the three risk factors independently associated with CVT development, as first proposed by $\operatorname{Virchow}^{23}$ in 1860. Need for ECMO, which occurred in $60 \%$ of the CTX patients, is itself associated with prolonged exposure to large caliber cannulas, non-pulsatile flow, and the coagulopathic state inherent to this modality.

The true incidence of CVTs is likely underrepresented in our study sample as screening evaluations for CVTs is not the standard of care in CTX patients. However, even small, otherwise hemodynamically insignificant CVTs could greatly increase the risk of CTX in the setting of elevated central venous pressures. This is supported by the fact that a majority $(>75 \%)$ of the patients who developed CTX in this study had single ventricle physiology and anatomy or other lesions commonly associated with elevated right heart pressures (i.e. complete AV Canal and Tetralogy of Fallot). Campbell, et al. ${ }^{7}$ reported a similarly elevated incidence of chylous effusions in this patient population. These high risk patients may justify screening evaluations for CVT development as a method of CTX prevention.

Our data further emphasize the need for prevention of CTX when considering the lack of an identifiable effect of the conservative treatment modalities on daily chylous effusate volumes. Octreotide, the synthetic analogue of the gastrointestinal hormone somatostatin, 
has been reported to reduce chylous output in multiple, small case series ${ }^{1-3,5}, 10,26,27,29-32$, 41, 42. In contrast, Landvoigt and Mullett ${ }^{41}$ demonstrated octreotide to be largely ineffective at reducing chylous chest tube output but did report a slight reduction in children with hypertriglyceridemia. As with many treatments, there is likely a reporting bias favoring positive results for octreotide therapy. However, the lack of any randomized, controlled studies evaluating the dosage, route of administration, or mechanism of action for octreotide results in inconsistent treatment regimens which could limit its effectiveness. More research is needed in this field.

We were surprised that MCT therapies did not exhibit a more clear benefit. By reducing that portion of the lymphatic circulation volume, it is reasonable to expect a significant reduction in drainage. As with octreotide, success with this therapy is limited to case reports ${ }^{24}$ and has never been studied in a randomized or controlled fashion. Anecdotally, however, we have had better outcomes with conservative treatment approaches in slightly older children (i.e. greater than three years old) but the younger, smaller infant is typically more severely affected by CTX and tends to require invasive approaches for definitive therapy.

The apparent lack of efficacy from conservative treatment approaches may be due to either a particularly resistant and heterogeneous sample population or ineffective treatments. These results should be interpreted with caution, however, as this retrospective study was neither powered nor designed to answer these questions. The different therapies were initiated at varying stages and hemodynamic states in each CTX patient's hospital course. Overlapping therapies (i.e. MCT formulas and octreotide) and 
various treatment dosages, durations and routes all limit the ability to statistically analyze these data.

Three infants in the CTX group received invasive procedures for their chylous effusions. One patient (TOF/ AVC, Trisomy 21) received bilateral pleuro-peritoneal shunts, which were removed after two months without incident. The remaining two patients both underwent unsuccessful thoracic duct ligation and ultimately died.

\section{$\underline{\text { Study Limitations }}$}

A universally accepted set of diagnostic criteria for CTX do not exist. This poses a significant issue for any retrospective evaluation of this entity. To our knowledge, the

only data specifically addressing this issue is from Buttiker, et al. ${ }^{43}$ Our pleural effusate labs are in keeping with their guidelines suggesting the diagnosis of CTX be made in the setting of a pleural TAG count of greater than $1.1 \mathrm{mmol} / \mathrm{L}(100 \mathrm{mg} / \mathrm{dL})$, pleural WBC count of greater than $1000 \mathrm{cells} / \mu \mathrm{L}$, and a lymphocyte predominance of greater than $80 \%$. As shown in Table 4, the standard deviations associated with our CTX group are large, which attests to the significant heterogeneity of these patients. Further, these guideline values are not indexed to serum values and do not account for present feeding status, somewhat limiting their utility. Until more stringent guidelines have been established, the diagnosis and appropriateness of therapy for CTX will always be subject to question. 


\section{Conclusions}

We have demonstrated that pediatric patients who develop CTX after surgery for congenital heart disease have a significantly increased incidence of and risk profile for CVT. The combination of this assertion with inadequate evidence for the efficacy of the available conservative treatment strategies urges the need for management strategies aimed at preventing the development of CTX. Prospective research into the possible causative role of CVT in CTX development is needed. 


\section{$\underline{\text { References }}$}

1. Al-Zubairy SA, Al-Jazairi AS. Octreotide as a Therapeutic Option for Management of Chylothorax. Ann Pharmacother. May 1, 2003;37(5):679-682.

2. Cheung Y-f, Leung MP, Yip M-m. Octreotide for treatment of postoperative chylothorax. The Journal of Pediatrics. July 2001;139(1):157-159.

3. Rimensberger PC, Muller-Schenker B, Kalangos A, Beghetti M. Treatment of a persistent postoperative chylothorax with somatostatin. The Annals of Thoracic Surgery. July 1998;66(1):253-254.

4. Nguyen DM, Shum-Tim D, Dobell AR, Tchervenkov CI. The management of chylothorax/chylopericardium following pediatric cardiac surgery: a 10-year experience. J Card Surg. July 1995;10(4 Pt 1):302-308.

5. Chan EH, Russell JL, Williams WG, Van Arsdell GS, Coles JG, McCrindle BW. Postoperative Chylothorax After Cardiothoracic Surgery in Children. Ann Thorac Surg. November 1, 2005;80(5):1864-1870.

6. Anton N, Massicotte MP. Venous thromboembolism in pediatrics. Semin Vasc Med. 2001;1(1):111-122.

7. Campbell RM, Benson LN, Williams WW, Adatia I. Chylopericardium after cardiac operations in children. Ann Thorac Surg. Jul 2001;72(1):193-196.

8. Petaja J, Lundstrom U, Sairanen H, Marttinen E, Griffin JH. Central venous thrombosis after cardiac operations in children. The Journal of Thoracic and Cardiovascular Surgery. October 1996;112(4):883-889.

9. Brissaud O, Desfrere L, Mohsen R, Fayon M, Demarquez JL. Congenital idiopathic chylothorax in neonates: chemical pleurodesis with povidone-iodine (Betadine). Arch. Dis. Child. Fetal Neonatal Ed. November 1, 2003;88(6):F531533.

10. Pratap U, Slavik Z, Ofoe VD, Onuzo O, Franklin RCG. Octreotide to treat postoperative chylothorax after cardiac operations in children. The Annals of Thoracic Surgery. November 2001;72(5):1740-1742.

11. Wolff AB, Silen ML, Kokoska ER, Rodgers BM. Treatment of refractory chylothorax with externalized pleuroperitoneal shunts in children. The Annals of Thoracic Surgery. September 1999;68(3):1053-1057.

12. Khosa JK, Leong SL, Borzi PA. Congenital cystic adenomatoid malformation of the lung: indications and timing of surgery. Pediatric Surgery International. July 2004;20(7):505-508.

13. Doerr CH, Allen MS, Nichols FC, Ryu JH. Etiology of chylothorax in 203 patients. Mayo Clinic Proceedings. 2005;80(7):867-870.

14. Manghat N, Hancock J, Walsh M, Puckett M, Noble R, Travis S. Thrombolysis for central venous occlusion causing bilateral chylothorax in a patient with Down syndrome. Journal of Vascular \& Interventional Radiology. 2004;15(5):511-515.

15. Fishman SJ, Burrows PE, Upton J, Hendren WH. Life-threatening anomalies of the thoracic duct: Anatomic delineation dictates management. Journal of Pediatric Surgery. August 2001;36(8):1269-1272.

16. Petaja J, Peltola K, Sairanen H, et al. Fibrinolysis, antithrombin III, and protein C in neonates during cardiac operations. J Thorac Cardiovasc Surg. September 1996;112(3):665-671. 
17. Moore RA, McNicholas KW, Naidech H, Flicker S, Gallagher JD. Clinically silent venous thrombosis following internal and external jugular central venous cannulation in pediatric cardiac patients. Anesthesiology. May 1985;62(5):640643.

18. Monagle P. Thrombosis in pediatric cardiac patients. Semin Thromb Hemost. December 2003;29(6):547-555.

19. Horigome H, Murakami T, Isobe T, Nagasawa T, Matsui A. Soluble P-selectin and thrombomodulin-protein C-Protein $\mathrm{S}$ pathway in cyanotic congenital heart disease with secondary erythrocytosis. Thromb Res. 2003;112(4):223-227.

20. Bernet-Buettiker V, Waldvogel K, Cannizzaro V, Albisetti M. Antithrombin activity in children with chylothorax. European Journal of Cardio-Thoracic Surgery. March 2006;29(3):406-409.

21. Monagle P, Chan A, Massicotte P, Chalmers E, Michelson AD. Antithrombotic therapy in children: the Seventh ACCP Conference on Antithrombotic and Thrombolytic Therapy. Chest. Sep 2004;126(3 Suppl):645S-687S.

22. Rich S. Clinical insights into the pathogenesis of primary pulmonary hypertension. Chest. September 1998;114(3 Suppl):237S-241S.

23. Makin A, Silverman SH, Lip GYH. Peripheral vascular disease and Virchow's triad for thrombogenesis. QJM. April 1, 2002;95(4):199-210.

24. Cormack BE, Wilson NJ, Finucane K, West TM. Use of Monogen for pediatric postoperative chylothorax. The Annals of Thoracic Surgery. January 2004;77(1):301-305.

25. Krafte-Jacobs B, Sivit CJ, Mejia R, Pollack MM. Catheter-related thrombosis in critically ill children: comparison of catheters with and without heparin bonding. $J$ Pediatr. January 1995;126(1):50-54.

26. Buck ML. Octreotide for the Management of Chylothorax in Infants and Children. University of Virginia's Pediatric Pharmacotherapy Monthly Newsletter. October 2004;10(10):1-4.

27. Rosti L, De Battisti F, Butera G, et al. Octreotide in the Management of Postoperative Chylothorax. Pediatric Cardiology. August 2005;26(4):440-443.

28. Buettiker V, Hug MI, Burger R, Baenziger O. Somatostatin: a new therapeutic option for the treatment of chylothorax. Intensive Care Med. Jun 2001;27(6):1083-1086.

29. Demos NJ, Kozel J, Scerbo JE. Somatostatin in the Treatment of Chylothorax. Chest. March 1, 2001;119(3):964-966.

30. Rosti L, Bini R, Chessa M, Butera G, Drago M, Carminati M. The effectiveness of octreotide in the treatment of post-operative chylothorax. European Journal of Pediatrics. March 2002;161(3):149-150.

31. Mohseni-Bod H, Macrae D, Slavik Z. Somatostatin analog (octreotide) in management of neonatal postoperative chylothorax: Is it safe? Pediatric Critical Care Medicine. 2004;5(4):356-357.

32. Markham KM, Glover JL, Welsh RJ, Lucas RJ, Bendick PJ. Octreotide in the treatment of thoracic duct injuries. Am Surg. December 2000;66(12):1165-1167. 
33. Milsom JW, Kron IL, Rheuban KS, Rodgers BM. Chylothorax: an assessment of current surgical management. J Thorac Cardiovasc Surg. Feb 1985;89(2):221227.

34. Ohtsuka T, Ninomiya M, Kobayashi J, Kaneko Y. VATS thoracic-duct division for aortic surgery-related chylous leakage. European Journal of Cardio-Thoracic Surgery. January 2005;27(1):153-155.

35. Pego-Fernandes PM, Jatene FB, Tokunaga CC, et al. Ligation of the thoracic duct for the treatment of chylothorax in heart diseases. Archives of Brasilian Cardiology. 2003;81(3):314-317.

36. Puntis J, Roberts K, Handy D. How should chylothorax be managed? Arch Dis Child. June 1, 1987;62(6):593-596.

37. Berkenbosch JW, Monteleone PM, Tobias JD. Chylothorax following apparently spontaneous central venous thrombosis in a patient with septic shock. Pediatric Pulmonology. 2003;35(3):230-233.

38. Dhande V, Kattwinkel J, Alford B. Recurrent bilateral pleural effusions secondary to superior vena cava obstruction as a complication of central venous catheterization. Pediatrics. Jul 1983;72(1):109-113.

39. Hanna J, Truemper E, Burton E. Superior vena cava thrombosis and chylothorax: relationship in pediatric nephrotic syndrome. Pediatric Nephrology. January 1997;11(1):20-22.

40. Kurekci E, Kaye R, Koehler M. Chylothorax and chylopericardium: A complication of a central venous catheter. The Journal of Pediatrics. June 1998;132(6):1064-1066.

41. Landvoigt MT, Mullett CJ. Octreotide efficacy in the treatment of chylothoraces following cardiac surgery in infants and children. Pediatr Crit Care Med. May 2006;7(3):245-248.

42. Tibballs J, Soto R, Bharucha T. Management of newborn lymphangiectasia and chylothorax after cardiac surgery with octreotide infusion. Ann Thorac Surg. Jun 2004;77(6):2213-2215.

43. Buttiker V, Fanconi S, Burger R. Chylothorax in Children: Guidelines for Diagnosis and Management. Chest. September 1, 1999;116(3):682-687. 


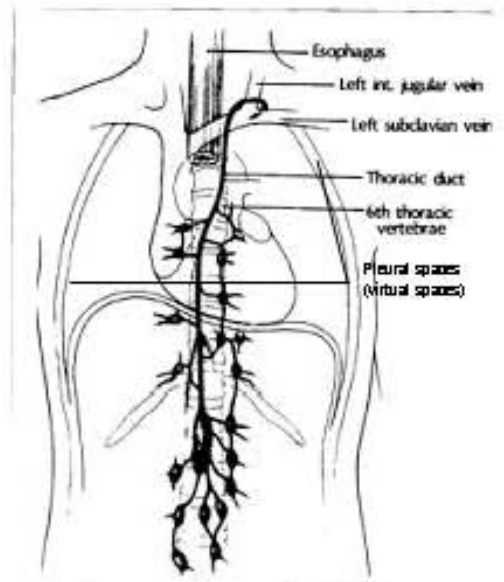

Figure 1. Thoracic Cavity 


\begin{tabular}{|cccc|}
\hline DEMOGRAPHIC VARIABLES & CTX & NON-CTX & P value/ Odds Ratio \\
\hline Admit Age in days (median) & $116.3 \pm 190.3(16)$ & $160.6 \pm 225.2(93)$ & $\mathbf{0 . 3 3}$ \\
Age at Surgery days (median) & $119.7 \pm 186.9(22)$ & $164.2 \pm 223.2(93)$ & $\mathbf{0 . 3 2}$ \\
Admit Weight in kg (median) & $4.67 \pm 2.63(3.48)$ & $5.21 \pm 2.81(4.66)$ & $\mathbf{0 . 1 5}$ \\
Vent Duration in days (median) & $31.9 \pm 36.8(21)$ & $6.1 \pm 5.9(4)$ & $\mathbf{0 . 0 0 5}$ \\
Total CPB Duration in minutes (median) & $89.5 \pm 79.4(101)$ & $81.9 \pm 69.6(84)$ & $\mathbf{0 . 5 9}$ \\
Total Number CXR (median) & $69.2 \pm 54(59)$ & $16.3 \pm 10.6(14)$ & $\mathbf{0 . 0 0 2}$ \\
Total Days NPO (median) & $23.1 \pm 20.9(22)$ & $5.9 \pm 4.4(4)$ & $\mathbf{0 . 0 0 5}$ \\
Total Days HAL (median) & $28.2 \pm 25.3(23.5)$ & $5.2 \pm 6.8(0)$ & $\mathbf{0 . 0 3}$ \\
ICU Duration in days (median) & $38.7 \pm 34.4(28.5)$ & $7.4 \pm 6.7(5)$ & $\mathbf{0 . 0 0 5}$ \\
Hospital Duration in days (median) & $52.1 \pm 34.2(49)$ & $16.8 \pm 14.4(10)$ & $<\mathbf{0 . 0 0 1}$ \\
Total Hospital Costs in Thousands (median) & $\$ 424 \pm 393(\$ 311)$ & $\$ 121 \pm 92(\$ 90)$ & $\mathbf{0 . 0 0 6}$ \\
Death (percent) & $7(23)$ & $3(5)$ & $\mathbf{6 . 2}(\mathbf{9 5 \%}$ CI 1.3-30.9) \\
\hline
\end{tabular}

Table 1. All continuous data are listed as mean \pm standard deviation. CXR- chest radiograph; ICUIntensive Care Unit; CPB- cardiopulmonary bypass; NPO- no enteral feeds; HAL- hyperalimentation 


\begin{tabular}{|c|c|c|}
\hline Anatomy & Surgery & Number \\
\hline Hypoplastic Left Heart Syndrome & Norwood/ Sano & 3 \\
\hline Hypoplastic Left Heart Syndrome & Hybrid Procedure & 1 \\
\hline Hypoplastic Left Heart Syndrome & Glenn Anastamosis & 3 \\
\hline Hypoplastic Right Ventricle & Damus-Kaye Stansel/ BT Shunt & 3 \\
\hline Severe Ebstein's & Starne's Procedure/ BT Shunt & 1 \\
\hline Hypoplastic Right Ventricle/ VSD & VSD Repair/ BT Shunt/ ASD Formation & 1 \\
\hline Tetralogy of Fallot & Complete Repair & 2 \\
\hline Tetralogy of Fallot/ Pulmonary Atresia & Complete Repair & 1 \\
\hline Tetralogy of Fallot/ Pulmonary Atresia & BT Shunt & 1 \\
\hline Tetralogy of Fallot/ Complete AV Canal & Tetralogy and AVC Repair & 3 \\
\hline Complete AV Canal & AV Canal Repair & 1 \\
\hline Coarctation of the Aorta & Extended End to End Reanastamosis & 3 \\
\hline Coarctation of the Aorta/ VSD & VSD and Coarct Repair & 1 \\
\hline Critical Aortic Stenosis/ Hypoplastic LV & Neonatal Heart Transplant & 1 \\
\hline Total Anomalous Pulmonary Venous Return & TAPVR Repair & 2 \\
\hline Partial Anomalous Pulmonary Venous Return & PAPVR/ ASD Repair & 1 \\
\hline Inlet type Ventricular Septal Defect & VSD Repair & 1 \\
\hline Partial AV Canal Defect & ASD Repair & 1 \\
\hline
\end{tabular}

Table 2. CTX Group description 


\begin{tabular}{|cccc|}
\hline & CTX & Non-CTX & $\begin{array}{c}\text { P Value/ } \\
\text { Odds Ratio }\end{array}$ \\
\hline Post-Operative ECMO (percent) & $18(60)$ & $5(8)$ & $\mathbf{9 . 9}$ (95\% CI 2.2-44.8) \\
Max CVP (median) & $15.3 \pm 3(14.9)$ & $14.3 \pm 3.5(13.6)$ & $\mathbf{0 . 0 5 8}$ \\
Min CVP (median) & $8.6 \pm 2.6(8.2)$ & $7.4 \pm 2.5(7.3)$ & $\mathbf{0 . 0 1 1}$ \\
Average CVP (median) & $12.0 \pm 2.7(11.6)$ & $10.8 \pm 2.9(10.4)$ & $\mathbf{0 . 0 1 9}$ \\
Days of UE CVC (median) & $12.2 \pm 10.4(11)$ & $7.1 \pm 9.8(4)$ & $\mathbf{0 . 0 6 7}$ \\
Days of LE CVC (median) & $13.0 \pm 14.9(9.5)$ & $7.1 \pm 9.2(3)$ & $\mathbf{0 . 0 6 2}$ \\
\hline
\end{tabular}

Table 3. All continuous data are listed as mean \pm standard deviation. ECMO- Extracorporeal Membrane Oxygenation; UE- Upper Extremity; LE- Lower Extremity; CVC- central venous catheter 


\begin{tabular}{|cc|}
\hline CTX Diagnostic Laboratory Data & Values \\
\hline Total Pleural WBC (median) & $2534 \pm 3990(870) / \mu \mathrm{L}$ \\
Pleural WBC Lymph \% (median) & $80.9 \pm 17.6(85)$ \\
Pleural TAG (median) & $413 \pm 724(233) \mathrm{mg} / \mathrm{dL}$ \\
\hline
\end{tabular}

Table 4. Data are expressed as mean \pm standard deviation. These values were all obtained on the day of CTX diagnosis or the day prior to diagnosis. 


\begin{tabular}{|cccc|}
\hline Lab Type & Pre CTX & Post CTX & P Value \\
\hline Total Protein (median & $4.8 \pm 0.8(4.8)$ & $4.7 \pm 0.7(4.6)$ & $\mathbf{0 . 0 7}$ \\
Albumin (median) & $3.5 \pm 0.7(3.5)$ & $3.3 \pm 0.7(3.3)$ & $\mathbf{0 . 0 5}$ \\
Serum TAG's (median) & $154 \pm 112(128)$ & $170 \pm 93(158)$ & $\mathbf{0 . 2 2}$ \\
Serum WBC (median) & $11.0 \pm 6.3(9.9)$ & $12.8 \pm 6.1(11.5)$ & $<\mathbf{0 . 0 0 0 1}$ \\
\hline
\end{tabular}

Table 5. Data are expressed as mean \pm standard deviation. These values apply to CTX subjects only and are the average of all values before and after date of CTX diagnosis, respectively. 